enorme Beträge erreicht. Von der zweiten Auffassung erklärt sich die Bewegung der Fixsterne so: die seitliche Tensorkomponente des Feldes, die auf der Erde als Corioliskraft beobachtet werden kann, treibt die Fixsterne im Kteise herum; ihre Feldstärke wächst proportional mit der Entfernung von der Erde, und darum erhalten die Fixsterne eine mit dem Abstand wachsende Rotationsgeschwindigkeit. Leteten Endes liegt hier also die gleiche Ursache vor, die wir in dem gleich schnellen Fallen aller Körper wirksam sehen, die Gleichheit von träger und schwerer Masse; die Fixsterne fallen frei in einem Gravitationsfeld, dessen Feldstarke mit dem Abstand von der Erde zunimmt. Daß aus Einsteizs Feldgleichungen tatshchlich solche Kräfte folgen, ist von Thirring gezeigt worden ${ }^{1}$ ).

3) Von hier aus klären sich auch die Einwănde Herrn Wulfs gegen die Äquivalenz des auf dem Karussell festen Koordinatensystems auf. Er glaubt, daß die von der Sonne ausgehenden Lichtstrahlen die Rotation der Welt gegen das Koordinatensystem nicht mitmachen, daß das Lichtbündel der Sonne relativ zum Karussell ruht und die Erde sich unter dem Lichtbündel wegdrehen mußte. Das ist aber der Irrtum, denn das tensorielle Gravitationsfeld packt ebenso die Lichtstrahlen, die ja Schwere haben, und dreht sie mit herum. Was die Sterne und die Erde dreht, ist eben dieses Gravitationsfeld; man darf nicht denken, daß diese Aufgabe dem Karusselpkerd zufallt. Die Kraft des Pferdes dient lediglich dazu, das Karussell davor zu bewahren, dem allgemeinen Zug zur Drehung zu folgen; das Pferd wirkt also wieder nur auf das Karussell, ganz wie in der gegenteiligen Auffassung. Man denke sich eine Fliege an der Wand eines Gefäßes, das im Beginn ist, herunterzufallen. Von der Gravitation gepackt, sinkt das Gefäß nach unten, und auch die Fliege wïrde infolge ihrer schweren Masse die Bewegung mitmachen.. Wenn sie aber mit entsprechender Geschwindigkeit an der Wand nach oben klettert, so vermag sie durch ihre Kraft das Gravitationsfeld zu kompensieren; aber ihre Anstrengung ist nur durch den Transport ihrer eigenen Masse bestimmt und ganz unabhängig von det Masse des Gefäßes. Auch wenn das Karussell still steht und man nur ein fingiertes Koordinatensystem rotieren laßst, ist das Gravitationsfeld da; Stuttgart, Physikal. Institut d. Techn. Hochschule, 192 I April.

1) Physikal. Zeitschrift 19.33, 19 I 8.

2) A. Kopff hat gezeigt, daß die Thirrixgschen Kräfte ebenfalls auf Massen zuräckgeführt werden können. Vergleiche auch die ausfuhrliche Darstellung des Rotationsproblems durch Kopff in Naturwiss. 9.9, $192 \mathrm{I}$.

\title{
Nordlichtbeobachtungen am 13. und 14. Mai 1921 in Bergedorf.
}

(Mitgeteilt von Prof, $R$. Schorr.)

In den beiden aufeinander folgenden Nachten vom I 3 . bis zum I5. Mai I92 1 warde auf der Hamburger Sternwarte in Bergedorf unter gâtustigen Bedingtungen ein Nordlicht von ungewöhnlich großer Entfalturg und Helligkeit beobachtet. Der Himmel war wolkenlos, die Sicht nach Norden für die Beobachter unbehindert; der Mond stand am Nachmittag des I 4. Mai' im ersten Viertel und ging in der ersten Nacht um $13^{\mathrm{h}}$, in der zweiten um $13 / 2$ tnter. Die Aufzeichmungen der einzelnen Beobachter (F. Dotberg, B. ALssow, R. Sckorr und $A$. Sikwaßmainn) sind im folgenden übersichtlich $z \mathrm{u}$ sammengestellt. Die Zeiten sind in Mittel-Europäischer Zeit angegeben. aber das Karussell folgt dem Zuge dann auch, weil die Pferdekraft es nicht zuruckhält. Und auch wenn das $\mathrm{Ka}$ ussell gleichförmig rotiert, das Pferd also abgesehen von Reibung keine Arbeit leistet, ist das Gravitationsfeld da und dreht fortwthrend die Sterne; mit der Leistung des Pferdes hat das Feld eben gar nichts zu tun.

Man wird noch einwenden: bei den Beispiel des fallenden Gefâes. ist das Gravitationsfeld durch die Erde erzeugt, aber woher stammt das Gravitationsfeld, das für das rotierende Koordinatensystem auftritt? Es stammt gerade so aus den Massen, wie jedes Gravitationsfeld ${ }^{2}$ ), nur erzeugen hier die rotierenden Massen selbst das Feld, das sie mitnimmt. Das ist nicht mehr paradox, sowie man dynamische Gravitationswirkungen annimmt. Auch eine bewegte elektrische Ladung erzeugt ein Feld, das sie selbst wieder steuert. Die Analogie ist sogar vollständig durchzuführen. Man denke sich ein Elektron, das in einem Inertialsystem ruht; von einem anderen Inertialsystem erscheint es dann gleichförmig bewegt. Von diesem System aus ist aber eine Kraft nötig, die die Ladung fortdrifckt; diese Kraft wird aus dem bemehr nötig ist.

4) Damit erledigt sich auch der letzte Einwand Herrn $W u l f \mathrm{~s}$, daß namlich Jahre vergehen müßten, bis die Antriebskraft des Karussels bei den Fixsternen angelangt ist und diese mitdreht. Nicht diese Kraft treibt die Sterne, sondern das Gravitationsfeld. Und dieses entsteht überall in den Massen selbst durch ihre Wechselwirkung und braucht nicht mehr Zeit zur Ubertragung als in der anderen Bewegungsauffassung auch.

5) Es erscheint von vornherein aussichtslos, durch solche Überlegungen die Relativitätstheorie widerlegen zu wollen. Denn sie ist ja gerade aus diesen begrifflichen Problemen herausgewachsen. Was man bezweifeln könnte, wäre, ob diese begrifflichen Forderungen auch in die mathematische Formulierung der Theorie eingegangen sind. Aber dies erscheint, nachdem Einstein die bekannten Gesetze der Himmelsmechanik, sogar noch um eine Stufe genauer, aus ihnen deduzieren konnte und Thirring und Kopff das Rotationsproblem aus ihnen lobsen konnten, kaum noch zweifelhaft.

\section{H. Reichenbach.}

\section{Nordlicht am 13. Mai r 921 . Gegen $9^{h} 55^{m}$ treten} tief am Nordhimmel einzelne kleine, helle, wolkenartige Gebilde von gelblicher Färbung in streifenförmiger Anordnung auf, die schnell auffeuchten und wieder verschwinden und trotz der noch herrschenden Abenddämmerung und des Mondscheins sich deutlich vom Himmel abheben. Die ersten senkrechten Strahlen steigen $10^{\mathrm{h}} 5^{\mathrm{m}}$ einzeln und auch in breiten Bandern tuber dem nordlichen Horizont empor und reichen bald bis zur Höhe des Polaris. $10^{\mathrm{h}}$ 1 $5^{\mathrm{m}-40^{\mathrm{m}}}$ spielt sich der glanzendste Teil der Erscheinung ab. Die wolkenartigen Gebilde nehmen an Anzahl, Helligkeit und Ausdehnung zu: es treten horizontal gerichtete Streifen, anfangs in etwa $20^{\circ} \mathrm{Höhe}$, 
auf, die an Intensität wechseln und bei ihrem Netterscheinen in immer größerer Höhe sichtbar werden. Die horizontalen Streifen treten hauptsächlich von $\mathrm{NW}$ bis $\mathrm{N}$ auf; außerdem erscheinen im NE einzelne Lichtwolken, getrennt von jenen. Besonders auffällig ist eine mächtige, vertikal aufsteigende, federförmige Wolke, deren Glanz so groß ist, daß die hellen Sterne im Cygnus nur noch schwach erscheinen und selbst Wega verblaßt. Die Szintillation dieser Wolken ist sehr lebhaft, ihre Färbung hellgrün, teilweise intensiv wie bengalisches Grünfeuer. Gegen $10^{\mathrm{h}} 30^{\mathrm{m}}$ überdecken die Lichtstreifen die ganze Nordhälfte des Himmels, zeitweise sogar bis zum Monde hin, und bis zum Zenit. Die Erscheinung erreicht $10^{\mathrm{h}} 3^{2^{\mathrm{m}}}$ ihren Höhepunkt; die Lichtstreifen liegen bis zy $10^{\circ}$ bis $20^{\circ}$ südlich vom Zenit. Der ganze nördliche Himmel ist wie von mächtigen Ci-Str-Streifen in ostwestlicher Streifung überflutet. Die. Helligkeit derselben bleibt nicht viel hinter derjenigen von Schäfchenwolken, die vom Monde bestrahlt sind, zurück. Die Lichtstreifen bieten im Verein mit den zwischen gelblichweiß und violett wechselnd, mitunter rötlich, gefärbten parallelen senkrechten Strahlen und Strahlenbüscheln eine überaus prächtige Erscheinung, der durch den am Westhimmel stehenden Mond nur wenig von ihrem Glanz genommen wird. Unterhalb des Mondes befinden sich auch noch zwei helle Lichtwolken. Am Nordhorizont erscheint der Himmelsgrund in leichtvioletter Färbung; die darüber hinschießenden senkrechten Strahlen sind nur schwach gefärbt. Einzelne Strahlen wandern seitlich von $W$ nach $E$. Bald darauf beginnt die Erscheinung zu erlöschen. Die wolkenartigen Gebilde verschwinden gegen $10^{h} 45^{\mathrm{m}}$, und es bleibt schließlich nur das helle Nordlichtsegment iiber einem sich breit von $\mathrm{W}$ bis $\mathrm{NE}$. über den Nordhorizont spannenden flachen dunklen Bogen von etwa $5^{\circ}$ Höhe übrig. Zwischen $\mathrm{II}^{\mathrm{h}}$ und $\mathrm{I}^{\mathrm{h}}$ tritt $\mathrm{zu}-$ weilen erneute, aber schwache Strahlenbildung im NNE auf. Das dunkle Segment ist $\left(\mathrm{I}^{\mathrm{h}} 3 \mathrm{O}^{\mathrm{m}}\right)$ nach oben hin sehr scharf und gleichmäßig hell begrenzt. Nach $I 2^{\mathrm{h}} 40^{\mathrm{m}}$ werden die Strahlen zahlreicher und heller und'erreichen von NW bis NE eine durchschnittliche Länge von $40^{\circ}$, ejnige reichen zeitweilig bis zu $55^{\circ}$ Höhe hinauf. Gegen $\mathrm{E} z u$ sind da, wo das Segment den Horizont erreicht, die Strahlungen scharf abgeschnitten. Auffällig ist, daß die Begrenzung des dunklen Segments nicht mehr so gleichmäßig und scharf ausgeprägt ist wie zuvor, sondern mit seiner unregelmäßig werdenden Form und tiefdunklen Färbung den Eindruck einer aufsteigenden Gewitterwand macht. $I 2^{\mathrm{h}} 50^{\mathrm{m}}$ ist die Gesamthelligkeit des Nordlichts so groß, daß das .Zifferblatt einer Taschenuhr deutlich abgelesen werden kann. Die Strahlen wandern seitlich von $W$ nach $E$, verblassen dann und sind als breiter heller Lichtschein mit senkrecht gerichteten Schattierungen bis $z u 40^{\circ}$ Höhe namentlich im NNW noch einige Zeit verfolgbar. I $3^{\mathrm{h}} 20^{\mathrm{m}}$ ist nur noch eine schwache Erhellung des Nordhimmels, aber weder Segment noch Strahlen zu sehen. $13^{\mathrm{h}} 35^{\mathrm{m}}-45^{\mathrm{m}}$ erscheinen zwei neue Strahlenbüschel: ein breites im NNW bei $\beta$ Aurigae, ein schmales im NNE im Perseus. I $3^{\mathrm{h}} 5^{0^{\mathrm{m}}}$ ist ein Streifen im NNW zu sehen. Dann wird die Morgendämmerung zu hell, um die Erscheinung weiter verfolgen zu können.

Nordlicht am I4. Mai r92I. Im NW bis $N$ werden von I I $^{\mathrm{h}} 23^{\mathrm{m}}$ ab bis $\mathrm{I}^{\mathrm{h}} 35^{\mathrm{m}}$ einige schwache Nordlichtstrahlen sichtbar, die bis $\mathrm{zu} 20^{\circ}$ Höhe aufschießen. Die Strahlen steigen nicht fächerförmig divergierend, sondern parallel senkrecht in drei Gruppen auf: östlich von Capella, hell und breit, westwärts wandernd, im Perseus dünn und fadenförmig, in der Cassiopeia bis über $\varepsilon$ Cass. hinaufragend. $12^{\mathrm{h}} 35^{\mathrm{m}}$ sind wieder einige schwache Nordlichtstrahlen, durch den Perseus gehend, im NNW für kurze Zeit sichtbar. $13^{\mathrm{h}} 6^{\mathrm{m}}$ erscheint ein dunkles Segment mit hellem Lichtsaum. Aufschießen starker, teilweise lila gefärbter Strahlen bis zu 40 bis $50^{\circ}$ Höhe besonders von NW bis N. $13^{\mathrm{h}} 16^{\mathrm{m}}$ erneutes Aufleuchten, zunächst scharf gegen $\mathrm{E}$ mit dem Perseus abschneidend, sodaß Cassiopeia freibleibt; dann setzt ein Wandern der Strahlen ostwärts ein. I $3^{\mathrm{h}} 2 \mathrm{O}^{\mathrm{m}}$ reicht der Strahlenvorhang bis Ursa major, breite still stehende Bänder. Die Helligkeit ist so groß, daß eine Taschenuhr abgelesen werden kann. Die Mitte des dunklen Segments liegt $\left(\mathrm{I} 3^{\mathrm{h}} 27^{\mathrm{m}}\right) \mathrm{im}$ Azimut weiter westlich als gestern und auch höher: der höchste Teil etwa bei $\beta$ Aurigae, sodaß der helle Saum diesen Stern beinahe erreicht. ${ }^{1} 3^{\mathrm{h}} 3^{6^{\mathrm{m}}}-40^{\mathrm{mi}}$ ist die Erscheinung außerordentlich glanzvoll. Breite teilweise vorhangartig erscheinende Lichtsäulen stehen unter Ursa major und unter Cassiopeia, besonders die letzteren sehr hell, erst weiß, dann violett, dann von rötlicher Färbung, die, mit schmalen scharf leuchtenden Strahlen durchsetzt, an Lebhaftigkeit zunimmt und in himbeerfarbige Tönung übergeht, während zwischen Auriga und Perseus Blaßgrün vorherrscht. Um $\times 3^{\mathrm{h}} 42^{\mathrm{ml}}$ verblaßt diese Erscheinung, aber gleich darauf treten neue intensive, genau senkrecht bis $70^{\circ}$ aufsteigende Strahlen im $\mathrm{N}$ auf. $13^{\mathrm{h}} 45^{\mathrm{m}}$ Ausdehnung des neuen Strahlengebietes von NNW bis NNE.' I $3^{\mathrm{h}} 47^{\mathrm{m}}$ geht die bis jetzt grünliche Färbung in lila über. Auch im NE erscheinen wieder sehr intensive lila Strahlen, sowie $13^{\mathrm{h}} 49^{\mathrm{m}}$ tief in NWzN horizontal gerichtete Lichtwolken und $13^{\mathrm{h}} 50^{\mathrm{m}}$ im WNW Strahlen oder Streifen, die schräg gegen $\mathrm{S}$ aufsteigen und im NE ein breites scharfbegrenztes, rötlich bis lila getöntes Band mit schmalen hellen Streifen darin. I $3^{\mathrm{h}} 59^{\mathrm{m}}$ verblassen die Strahlen im NE, aber im NW jetzt intensive grünliche Strahlen. $\quad 4^{\mathrm{h}} 6^{\mathrm{m}}$ erneute Bildung eines dunklen Segments, das flacher und niedriger als um $13^{\text {h }} 6^{\mathrm{m}}$ ist und aus dem $\mathrm{I} 4^{\mathrm{h}} 7^{\mathrm{m}}$ im NNE und $\mathrm{N}$ neue helle und scharfe Strahlen, unten grünlich, oben lilafarben, emporschießen. Nach plötzlichem Abflauen und Verlöschen der ganzen Erscheinung erfolgt $14^{\mathrm{h}} 9^{\mathrm{m}}-20^{\mathrm{m}}$ ein neues Aufleuchten einer ganz neuen Lichtverteilung über dem dunklen Segment von $W$ bis NNE, deren Mitte im NW liegt und die trotz Morgendämmerung noch gut sichtbar ișt.

Hamburger Sternwarte, I 92 I Mai.

R. Schorr.

Inhalt zu Nr. 5107. W. Heiskanen, H. Ludendorff. Über die Radialgeschwindigkeiten der Mira Ceti-Sterne. 297, - P: Lenard. Fragen der Lichtgeschwindigkeit. 303. - H. Reichenbach. Erwiderung auf Herrn Th. Wulfs Einwände gegen die allgemeine Relativitätslehre. 307. - R. Schorr. Nordlichtbeobachtungen am 13. und I4. Mai 1921 in Bergedorf. 309. 